\title{
Preface: Nitric Oxide and Cancer: Pathological and Therapeutic Aspects
}

Recently, there have been many advances in the investigation of the controversial roles that nitric oxide (NO) plays in various human cancers. These opposing roles as protumorigenic and antitumorigenic have been reported in the literature in various preclinical models. In general, international conferences on NO have focused primarily on its role in noncancer disease manifestations. Therefore, it became apparent that a more focused workshop on the role of NO in cancer was warranted, and so a series of workshops were initiated. The first was held in Paris, France; the second, in Kyoto, Japan; the third, in Kingston, Ontario, Canada; and the fourth, in Sevilla, Spain. The fifth workshop, in 2017 was held in Bologna, Italy and was organized by the guest editors of this issue, Dr. Greta Varchi and Dr. Valentina Rapozzi.
This special issue deals with these proceedings and covers several topics on the various roles that NO and its metabolites play in various cancers along with underlying mechanisms of action. Contrasting activities mediated by NO in cancer were presented.

\section{Guest Editors:}

\section{Valentina Rapozzi}

Department of Medical Sciences

University of Udine

Udine, Italy

\section{Greta Varchi}

Italian National Research Council

Institute of Organic Synthesis and Photoreactivity

Bologna, Italy 
